\title{
Locking and noise properties of multisection semiconductor lasers with optical injection Application to Fabry-Perot and DFB cavities
}

Fabien Kéfélian and Philippe Gallion, Senior Member, IEEE,

\begin{abstract}
An analytical theory is presented for the study of injection locking in multisection semiconductor lasers. The Helmoltz equation for the electric field is solved using the Green's function method and the injected fields are included via the boundary conditions. Two cases are distinguished, injection through the front facet of the laser and injection through the rear facet. In both cases, an equation of evolution for the envelope of the electric field is established, taking into account the longitudinal distribution of the carrier and photon densities and the nonlinear gain. The expressions of the intensity, phase and carrier density noise spectra are derived using a matrix formulation. Comparison to classical equations used for Fabry-Perot lasers is discussed. The locking properties of a DFB laser with an anti-reflection coated front facet are studied in detail. Results demonstrate the strong sensitivity of the locking properties on the phase grating and rear facet reflectivity.
\end{abstract}

\section{Index Terms}

Injection locked oscillators, distributed feedback lasers, optical noise, semiconductor lasers

F. Kéfélian was with the Département Communications et Électronique, École Nationale Supérieure des Télécommunications, Paris, France. He is now with the Laboratoire de Physique des Lasers, Université Paris-XIII, Villetaneuse, France

P. Gallion is with the Département Communications et Électronique, École Nationale Supérieure des Télécommunications, Paris, France

The École Nationale Supérieure des Télécommunications is part of the Groupe des Écoles de Télécommunications and its research activities are associated with the Centre National de la Recherche Scientifique, France 



\section{Locking and noise properties of multisection semiconductor lasers with optical injection}

\section{Application to Fabry-Perot and DFB cavities}

\section{INTRODUCTION}

Optical injection locking is a powerful technique to improve the performance of diode lasers and realize new techniques in telecommunications [1] [2] [3] [4] [5]. It is also a very interesting phenomenon in terms of physics and non-linear dynamics [6] [7] [8] [9] and can be used as a tool for determining fundamental laser parameters [10] [11]. Optical injection locking was first experimentally demonstrated more than forty years ago in gas lasers [12] and its study in semiconductor lasers started in the early 1980's [13] [14] (see [15] for an extended bibliography on the history of optical injection). Contrary to conventional lasers, semiconductor lasers allow a significant fraction of the light to escape and are consequently more sensitive to external perturbations. Moreover, the important dependence of the refractive index on the carrier density in semiconductor lasers has been shown to significantly affect the injection locking properties [16]. The first works on injection locking in semiconductor lasers were mainly dedicated to the study of the range of locking, the stability [17] [18], and the noise properties [19] [20] [21]. Their results have demonstrated the interest of this technique for frequency stabilization and linewidth narrowing. The analyze of the modulation properties of a locked diode laser has shown the possibility to reduce the relaxation oscillations [22] [23], extend the modulation bandwidth, generate phase modulation and to reduce the chirp [21] [24] [25]. The influence of the gain compression has been also investigated [26] and has been shown to be a factor contributing to stabilization of the locking [27].

Optical injection in semiconductor lasers has been mainly studied using simple rate equations for the outgoing electric field and the carrier density. Some authors have derived these rate equations from the full wave-equation [28] [29], or the round trip time traveling wave amplifier model [15], but many works have used a modified version of the rate equation for isolated diode laser by phenomenologically introducing the external field with a coupling factor or feed-in rate. Consequently, the expression of the feed-in rate has sometimes not been set clearly or justified correctly. Moreover, for lasers exhibiting spatial hole burning or containing Bragg gratings, such as DFB or DBR lasers, rate equations cannot be derived without first using a full longitudinal treatment. DFB lasers with external feedback have been the subject of several theoretical studies [30] [31], but few results have been published concerning injection locking in DFB lasers. Experimental studies [32] [33] [34] have for example demonstrated that, contrary to a Fabry-Perot laser, a DFB laser exhibits a symmetric locking range for weak injection, due to the possibility of having a stable oscillation in a mode with higher threshold gain than the free mode. However, an investigation of the locking range as a function of the DFB laser fundamental parameters is still missing. 
Different mathematical tools can be used for the full longitudinal treatment of a multisection laser [35] [36] [37] [38] [39] [40]. Among them, the Green's function method is well appropriate to derive analytical expressions. The Green's function method was introduced by C. Henry [41] to analyze the spontaneous emission of semiconductor amplifiers and multisection lasers. This work triggered numerous successful studies concerning the dynamics and noise properties of DFB and more generally of multisection lasers [42] [43] [44] [45] [46]. B. Tromborg et al. used this method to study the effect of spatial hole burning, nonuniform current injection and nonlinear gain on the linewidth of DFB lasers [47], as well as analyzing the stability and the noise and modulation properties [48] [49] [50]. The coherence collapse conditions were also derived using the same formalism [51]. The influence of the structure of multisection lasers on the phase-amplitude coupling factor and the spontaneous emission rate have been studied with Green's function by Duan et al. [52]. An effective phase-amplitude coupling parameter has been defined and including the non linear gain new explanations to the rebroadening of the spectrum at high power has been given. More recently, Green's function has been used for new studies of the external optical feedback, concerning the threshold of the coherence collapse [53] and the spectrum of external cavity lasers [54].

In this paper, we present a theoretical study of the injection locking in a multisection laser. This work extends a previous work of B. Tromborg et al. [31] which used the concept of effective coefficients of reflection and transmission. Two questions are at the source of our investigation: For DFB lasers, which "round trip time" (or which $Q$ factor) can be used for the expression of the feed-in rate? For symmetric lasers, is there a difference between an injection through the facet from which the emission is observed (front facet), and an injection through the rear facet? The purpose of the paper is to obtain a general equation of motion for the electric field envelope in both cases of injection through the front and rear facet, and to hereby derive the noise properties of the injection locked laser.

The paper is organized in the following way, in Section II, starting from the equation of propagation and using the Green's function method, we derive an expression for the Fourier transform of the electric field at the output of the laser as a function of the external injected fields to both facets and a Langevin force representing spontaneous emission. The characteristics of the laser are taken into account using effective reflection and transmission coefficients. In Section III and IV, the equation of motion of the temporal complex envelope of the electric field is calculated, using a Taylor expansion of the effective coefficients of the cavity, for respectively backward and forward injection. For each direction, the rate equations are linearized to give the locking relation and to determine the power spectral densities of the noises in matrix form. In Section V we apply our results, concerning the feed-in rate value, to the Fabry-Perot cavity and discuss the comparison to the classical expression of R. Lang. After, the parameter of injection for a DFB laser with an output facet anti-reflection coated are numerically studied. Finally, the conclusions are summarized in Section VI.

\section{ELECTRIC FIELD IN MULTISECTION LASER WITH EXTERNAL INJECTION}

Fig. 1. Laser submited to the injection by both facets 
We consider the general configuration of Fig. 1.The section between $z=0$ and $z=l$ represents the laser (Fabry-Perot, DFB or multisections). The internal electric field $\vec{E}$ is decomposed into right and left traveling fields $\vec{E}^{+}$and $\vec{E}^{-}$. At each interface we define a reflection and two transmission coefficients: at $z=0, r_{1}, t_{12}$ (into the laser) and $t_{21}$ (out of the laser); at $z=l, r_{2}, t_{23}$ (out of the laser) and $t_{32}$ (into the laser). The laser can generally emit light via both facets, but all along the paper we consider that only the field $\vec{E}_{\text {out }}^{+}$emitted through the right facet $(z=l)$ is observed and studied. The right facet is consequently called the front facet, and the left facet is called the rear facet. We assume that an electric field $\vec{E}_{i n j R}^{-}$can be injected through the front facet, using a coupler or a circulator, or that an electric field, $\vec{E}_{i n j L}^{+}$, can be injected through the rear facet.

The starting point is the equation of propagation of the electric field established using the Maxwell's equations in a macroscopic medium:

$$
\vec{\nabla}^{2} \vec{E}(\vec{r}, t)-\mu_{0} \sigma \frac{\partial \vec{E}}{\partial t}(\vec{r}, t)-\frac{1}{c^{2}} \frac{\partial^{2} \vec{E}}{\partial t^{2}}(\vec{r}, t)=\mu_{0} \frac{\partial^{2} \vec{P}}{\partial t^{2}}(\vec{r}, t)
$$

with $\vec{r}=(x, y, z)$ the position, $\vec{P}$ the electric polarization, $\mu_{0}$ and $\epsilon_{0}$ the magnetic permeability and electric permitivity of the vacuum, $c$ the light velocity, and $\sigma$ the conductivity. $\vec{P}$ can been expressed as a function of the electric field introducing the susceptibility $\chi$. The spontaneous electric polarization, due to quantification of the field, is included using a random function, $\vec{P}_{s}$ :

$$
\vec{P}(\vec{r}, t)=\epsilon_{0} \chi(\vec{r}, t) * \vec{E}(\vec{r}, t)+\vec{P}_{s}(\vec{r}, t)
$$

Susceptibility and conductivity can be taken into account together through the relative dielectric constant $\epsilon_{r}$ :

$$
\epsilon_{r}(\vec{r}, \omega)=1+\chi(\vec{r}, \omega)-j \frac{\sigma(\vec{r}, \omega)}{\epsilon_{0} \omega}
$$

with $\omega$ the Fourier angular frequency.

The Fourier transform of the electric field is a solution of the Helmoltz equation:

$$
\vec{\nabla}^{2} \vec{E}(\vec{r}, \omega)+\frac{\omega^{2}}{c^{2}} \epsilon_{r}(\vec{r}, \omega) \vec{E}(\vec{r}, \omega)=\vec{F}(\vec{r}, \omega)
$$

with $\vec{F}(\vec{r}, \omega)=-\omega^{2} \mu_{0} \vec{P}_{s}(\vec{r}, \omega) . \vec{F}$ is a Langevin force whose correlation coefficient is given by the theorem of fluctuation dissipation [55]:

$$
\begin{aligned}
\left\langle\vec{F}\left(\overrightarrow{r^{\prime}}, \omega\right) \vec{F}^{*}\left(\vec{r}, \omega^{\prime}\right)\right\rangle & =2 D_{\vec{F} \vec{F}^{*}} \delta\left(\vec{r}-\vec{r}^{\prime}, \omega-\omega^{\prime}\right) \\
D_{\vec{F} \vec{F}^{*}}(\vec{r}, \omega) & =\frac{2 \pi \omega^{3} \hbar}{c^{3} \epsilon_{0}} n_{m}(\vec{r}) g_{m}(\vec{r}) n_{s p} \\
n_{s p} & =\frac{1}{1-e^{\left(\frac{\hbar \omega-E_{c v}}{k_{B} T}\right)}}
\end{aligned}
$$

with $T$ the temperature, $k_{B}$ the Boltzmann constant, $h$ the Planck constant, $\hbar=h / 2 \pi, E_{c v}$ the energy difference between the quasi Fermi levels, $n_{m}=\sqrt{\Re e\left\{\epsilon_{r}\right\}}$ the material refractive index, $g_{m}=\frac{\omega}{c n_{m}} \Im m\{\chi\}$ the material gain, and $n_{s p}$ the inversion factor. 
We assume that, in each section $i$ of the laser, the electric field oscillates in the fundamental transverse mode $\Phi_{i}(x, y)$ polarized along $\vec{u}$ with the complex constant of propagation $k_{i}(z, \omega)$ satisfying [56]:

$$
\left[\frac{\partial^{2}}{\partial x^{2}}+\frac{\partial^{2}}{\partial y^{2}}+\frac{\omega^{2}}{c^{2}} \epsilon_{r}(x, y, z, \omega)\right] \Phi_{i}(x, y)=k_{i}^{2}(z, \omega) \Phi_{i}(x, y)
$$

Consequently $\vec{E}(\vec{r}, \omega)$ can be written as $E(z, \omega) \Phi_{i}(x, y) \vec{u}$ with $E(z, \omega)$ satisfying:

$$
\left[\frac{\partial^{2}}{\partial z^{2}}+k^{2}(z, \omega)\right] E(z, \omega)=f(z, \omega)
$$

where

$$
f(z, \omega)=\frac{\iint \vec{F}(x, y, z, \omega) \Phi(x, y) \vec{u} d x d y}{\iint \Phi(x, y) \Phi(x, y) d x d y}
$$

represents the spontaneous emission in the transverse mode.

Solving the inhomogeneous differential equation (9) using the Green's function method (Appendix A), the Fourier transform of the forward traveling component of the electric field at the front facet of the laser can be expressed as a function of the injected fields and a Langevin force:

$$
E^{+}\left(l^{-}, \omega\right)=\frac{t_{L} t_{12} E_{i n j L}^{+}\left(0^{-}, \omega\right)+r_{L} t_{32} E_{i n j R}^{-}\left(l^{+}, \omega\right)+F_{L}}{\left[1-r_{2} r_{L}\right]}
$$

where $r_{L}$ and $t_{L}$ are the equivalent reflection and transmission coefficients of the active region between $z=0^{-}$ and $z=l^{-}$for a backward traveling wave and $F_{L}$ is a Langevin force representing the total spontaneous emission at the output of the laser. Expressions of $r_{L}, t_{L}$ and $F_{L}$ are derived in Appendix A.

\section{DYNAMIC EQUATIONS AND NOISE PROPERTIES OF LASER DIODE WITH BACKWARD INJECTION}

We consider in this section the injection of a backward traveling wave $\vec{E}_{i n j R}^{-}$through the front facet, i.e. backward injection.

\section{A. Expansion of the electric field equation}

For injection through the front facet (11) becomes:

$$
\frac{E^{+}\left(l^{-}, \omega\right)}{r_{L}}=r_{2} E^{+}\left(l^{-}, \omega\right)+t_{32} E_{i n j R}^{-}\left(l^{+}, \omega\right)+\frac{F_{L}(\omega)}{r_{L}}
$$

$r_{L}$ depends on $\omega$, but also on the carrier and photon densities $\mathcal{N}(z)$ and $\mathcal{P}(z)$, via $\epsilon_{r}$. Since $\mathcal{N}$ and $\mathcal{P}$ depend on $z, r_{L}$ is a functional, i.e. a function of functions.

$\mathcal{P}(z)$ is the transversely average photon density in the active layer (A.L.):

$$
\mathcal{P}(z)=\frac{2 \epsilon_{0} \mathrm{nn}_{\mathrm{g}_{c a}}(z) n_{g m} \Gamma}{\hbar \omega_{0} A_{a c t}}|E(z)|^{2}
$$

where $\operatorname{nn}_{\mathrm{g}_{c a}}(z)$ is the value of the product of the material refractive index and the group refractive index $n_{g m}=$ $n_{m}+\omega \partial n_{m} / \partial \omega$ transversally average on the active layer:

$$
\operatorname{nn}_{\mathrm{g}_{c a}}(z)=\frac{1}{A_{a c t}} \iint_{A . L .} n_{m}(\vec{r}) n_{g m}(\vec{r})|\Phi(x, y)|^{2} d x d y
$$


$\Gamma$ is the confinement factor of the transverse mode in the active layer and $A_{a c t}$ the transverse area of the active layer [56].

Without injection, when spontaneous emission is neglected, the static solution $\left(\omega_{s}, \mathcal{N}_{s}(z), \mathcal{P}_{s}(z)\right)$ fulfills the condition of oscillation:

$$
r_{2} r_{L}\left(\omega_{s}, \mathcal{N}_{s}(z), \mathcal{P}_{s}(z)\right)=1
$$

When the laser is submitted to optical injection, the static parameters of the laser are modified. Once locking is achieved, the central frequency of the slave laser becomes equal to the central frequency of the master laser $\omega_{i}$. Assuming that the function $1 / r_{L}$ is slowly varying in a region around $\left(\omega_{s}, \mathcal{N}_{s}, \mathcal{P}_{s}\right)$, and that the detuned state with deviation $\Delta \mathcal{N}$ and $\Delta \mathcal{P}$ is included in this region, the value of $1 / r_{L}$ can be determined using a Taylor expansion:

$$
\begin{aligned}
\frac{1}{r_{L}(\omega, \mathcal{N}, \mathcal{P})} \approx & \frac{1}{r_{L s}}+\left[\frac{\partial \frac{1}{r_{L}}}{\partial \omega}\right]_{s}\left(\omega-\omega_{s}\right) \\
& +\int_{0}^{l}\left[\frac{\delta \frac{1}{r_{L}}}{\delta k}(z) \frac{\partial k}{\partial \mathcal{N}}\right]_{s}(\Delta \mathcal{N}(z)) d z \\
& +\int_{0}^{l}\left[\frac{\delta \frac{1}{r_{L}}}{\delta k}(z) \frac{\partial k}{\partial \mathcal{P}}\right]_{s}(\Delta \mathcal{P}(z)) d z
\end{aligned}
$$

$\delta 1 / r_{L}$ is the functional derivate of $1 / r_{L}$ introduced by B. Tromborg et al. in [47].

$$
\delta \frac{1}{r_{L}}=\int_{0}^{l} \frac{\delta \frac{1}{r_{L}}}{\delta k}(z) \delta k(z) d z
$$

where $\delta\left(1 / r_{L}\right) / \delta k$ represents the infinitesimal variation of $1 / r_{L}$ due to an infinitesimal variation $\delta k$ of the function $k$.

\section{B. Temporal envelope of the electric field}

The complex envelope around $\omega_{i}$ of the forward traveling component of the intracavity electric field at the front facet is defined by:

$$
A^{+}(t)=\frac{1}{2 \pi} \int_{0}^{\infty} E^{+}\left(l^{-}, \omega\right) e^{j\left(\omega-\omega_{i}\right) t} d \omega
$$

The envelope of the injected field, $A^{i}(t)$, is defined similarly in $l^{+}$.

Using the expansion of $1 / r_{L}$ in the equation (12), the equation of motion of $A^{+}$can be obtain by inverse Fourier transform:

$$
\begin{aligned}
\frac{d A^{+}}{d t}(t)= & -j\left(\omega_{s}-\omega_{i}\right) A^{+}(t) \\
& +A^{+}(t)\left\langle C_{\mathcal{N}} \mid \Delta \mathcal{N}(t)\right\rangle \\
& +A^{+}(t)+\left\langle C_{\mathcal{P}} \mid \Delta \mathcal{P}(t)\right\rangle \\
& +f_{R} t_{32} A^{i}(t) \\
& +F_{A}(t)
\end{aligned}
$$


with

$$
\begin{gathered}
f_{R}=r_{L s} f_{D}=j\left(\frac{\partial \frac{1}{r_{L}}}{\partial \omega}\right)_{s}^{-1} \\
C_{\mathcal{N}}(z)=-\left[\frac{\delta \frac{1}{r_{L}}}{\delta k}(z) \frac{\partial k}{\partial \mathcal{N}}\right]_{s} f_{R} \\
C_{\mathcal{P}}(z)=-\left[\frac{\delta \frac{1}{r_{L}}}{\delta k}(z) \frac{\partial k}{\partial \mathcal{P}}\right]_{s} f_{R}
\end{gathered}
$$

We use here a notation similar to the notation of Dirac in quantum mechanics:

$$
\langle f \mid g\rangle=\int_{0}^{l} f\left(z^{\prime}\right) g\left(z^{\prime}\right) d z^{\prime}
$$

The derivates of $k$ are given by:

$$
\begin{aligned}
\frac{\partial k}{\partial \omega} & =\frac{1}{v_{g}}+\frac{j}{2} \frac{\partial g}{\partial \omega} \\
\frac{\partial k}{\partial \mathcal{N}} & =j \frac{1+j \alpha_{H}}{2} \frac{\partial g}{\partial \mathcal{N}}
\end{aligned}
$$

with $\alpha_{H}=-2 k \frac{\partial n / \partial \mathcal{N}}{\partial g / \partial \mathcal{N}}$ the Henry factor, $g$ the modal gain, $n$ the modal refractive index and $v_{g}$ the group velocity.

The first term on the right hand side of (19) represents the rotation of $A^{+}$due to the detuning, the second and third ones represent the in phase and in quadrature contributions due to the modification of the gain and index induced by the carrier and photon density modifications. The functions $C_{\mathcal{N}}$ and $C_{\mathcal{P}}$ determine the variation of the global gain and index of the cavity due to the local variation in $z$ of the carrier and photon densities. They take into account longitudinal spatial hole burning [57] and spectral hole burning [58]. The fourth term represents the contribution of the injected field. $F_{A}$ is the Langevin force representing the contribution of the spontaneous emission to the envelope evolution:

$$
F_{A}(t)=\frac{f_{D}}{2 \pi} \int_{0}^{\infty} F_{L}(\omega) e^{j\left(\omega-\omega_{i}\right) t} d \omega
$$

The autocorrelation of $F_{A}$ is derived from (6), (10), (26) and (98) (Appendix A):

$$
\left\langle F_{A}(t) F_{A}^{*}\left(t^{\prime}\right)\right\rangle=\frac{R_{s p}}{\sigma_{p}^{2}} \delta\left(t-t^{\prime}\right)
$$

with

$$
R_{s p}=\frac{4 \omega_{0}^{3} \hbar\left\langle\left.\mathrm{nn}_{\mathrm{g}}|| Z_{L}\right|^{2}\right\rangle\left|f_{D}\right|^{2}\left\langle\left.\mathrm{ng} n_{s p}|| Z_{L}\right|^{2}\right\rangle\left|Z_{1}^{+}(0)\right|^{2}}{c^{3}\left|\iint \Phi(x, y) \Phi(x, y) d x d y\right|^{2}\left|Z_{2}^{-}(l)\right|^{2}}
$$

where the $Z$ functions are defined in Appendix A. $R_{s p}$ is the average rate of spontaneous emission into the mode, taking into account the effect of the transverse mode [59] and the longitudinal mode [41] [47] [31] on the standard relation of Einstein $R_{s p}=v_{g} g n_{s p} . \sigma_{p}$ is a constant such that $p=\sigma_{p}^{2}|A|^{2}$ is the total number of photons in the laser cavity:

$$
\sigma_{p}^{2}=2 \frac{\epsilon_{0}}{\hbar \omega_{s}} \int \operatorname{nn}_{\mathrm{g}}(z)\left|Z_{L}(z)\right|^{2} d z
$$

with

$$
\operatorname{nn}_{\mathrm{g}}(z)=\iint n(\vec{r}) n_{g}(\vec{r})|\Phi(x, y)|^{2} d x d y
$$


and

$$
\operatorname{ng}(z)=\iint n_{m}(\vec{r}) g_{m}(\vec{r})|\Phi(x, y)|^{2} d x d y
$$

\section{Optical phase and power equations}

The mean optical power $I$ emerging from the front facet of the laser is given by:

$$
I(t)=T_{2} \sigma_{I}^{2}\left|A^{+}(t)\right|^{2}
$$

where $\sigma_{I}^{2}=2 \epsilon_{0} c n_{g}\left(l^{-}\right), n_{g}$ is the modal group refractive index and $T_{2}=1-\left|r_{2}\right|^{2}$. The incident optical power $I_{\text {in }}(t)$ is similarly given by:

$$
I_{\text {in }}(t)=\frac{\sigma_{I}^{2}}{n_{g}\left(l^{-}\right)}\left|A_{\text {in }}(t)\right|^{2}
$$

The rate equations for the output power $I(t)$ and the phase of $A^{+}, \phi(t)$, are determined by multiplication of (19) by $4 A^{+*} \epsilon_{0} c n_{g}\left(l^{-}\right) T_{2}$ and separation into real and imaginary parts:

$$
\begin{aligned}
\frac{d I}{d t}(t)= & 2 I(t)\left\langle\Re e\left\{C_{\mathcal{N}}\right\} \mid \Delta \mathcal{N}(t)\right\rangle \\
& +2 I(t)\left\langle\Re e\left\{C_{\mathcal{P}}\right\} \mid \Delta \mathcal{P}(t)\right\rangle \\
& +2 T_{2} \sqrt{I(t) I_{\text {in }}(t)}\left|f_{R}\right| \cos \left(\theta(t)+\psi_{R}\right) \\
& +F_{I}(t) \\
\frac{d \phi}{d t}(t)= & \left(\omega_{s}-\omega_{i}\right)+\left\langle\Im m\left\{C_{\mathcal{N}}\right\} \mid \Delta \mathcal{N}(t)\right\rangle \\
& +\left\langle\Im m\left\{C_{\mathcal{P}}\right\} \mid \Delta \mathcal{P}(t)\right\rangle \\
& +T_{2} \sqrt{\frac{I_{\text {in }}(t)}{I(t)}}\left|f_{R}\right| \sin \left(\theta(t)+\psi_{R}\right) \\
& +F_{\phi}(t)
\end{aligned}
$$

where $\theta(t)=\phi_{i n}(t)-\phi(t), \phi_{i n}$ is the phase of $A^{i}$ and $\psi_{R}=\arg \left\{f_{R}\right\}$.

The carrier density is governed by the rate equation:

$$
\begin{aligned}
\frac{d \mathcal{N}(z, t)}{d t}= & J(z, t)-\mathrm{R}(\mathcal{N}(z, t)) \\
& -v_{g} g(\mathcal{N}(z, t), \mathcal{P}(z, t)) \mathcal{P}(z, t) \\
& +F_{\mathcal{N}}(z, t)
\end{aligned}
$$

where $J$ is the density of injected carriers, $\mathrm{R}$ is the function describing the spontaneous recombination of the carriers and $F_{\mathcal{N}}(z, t)$ is the Langevin force associated to the fluctuation of carriers due to the interaction between the field and the spontaneous electric polarization [60]. 
The non zero Langevin forces diffusion coefficients are [61]:

$$
\begin{aligned}
2 D_{I I} & =2 R_{s p} I_{0} T_{2}\left(\frac{\sigma_{I}}{\sigma_{p}}\right)^{2} \\
2 D_{\phi \phi} & =\frac{R_{s p}}{2 p_{0}} \\
2 D_{\mathcal{N N}}(z) & =2 \frac{\left[v_{g} g_{0}(z) n_{s p} \mathcal{P}_{0}(z)+\mathrm{R}(\mathcal{N}(z))\right]}{A_{a c t}} \\
2 D_{I \mathcal{N}}(z) & =-2 v_{g} g_{0}(z) n_{s p} \mathcal{P}_{0}(z) T_{2}\left(\frac{\sigma_{I}}{\sigma_{p}}\right)^{2}
\end{aligned}
$$

\section{Calculation of the locking range}

Locked states $\left(\theta_{0}, I_{0}, \mathcal{N}_{0}(z), \mathcal{P}_{0}(z)\right)$ are solutions of (34), (35) and (36) with all noise and derivative terms set to zero. When locking is achieved, the phase difference and angular frequency detuning are related by:

$$
\omega_{i}-\omega_{s}=\eta_{b}\left|f_{R}\right| \sqrt{1+\alpha_{e f f}^{2}} \sin \left(\theta_{0}+\psi_{R}-\tan ^{-1}\left(\alpha_{e f f}\right)\right)
$$

where $\eta_{b}=\sqrt{\frac{I_{i n 0} T_{2}}{I_{0} / T_{2}}}, \eta_{b}^{2}$ is the ratio between the optical power injected through the front facet and the forward traveling intracavity optical power at the front facet. It is important to point out here that, for a locked state, it is possible to define a stationary mean value of the difference of the slave and master phases, but not of the individual phases which remain unstationary random functions. A generalized Henry factor has been used:

$$
\alpha_{e f f}=\frac{\left\langle\Im m\left\{C_{\mathcal{N}}\right\} \mid \Delta \mathcal{N}\right\rangle+\left\langle\Im m\left\{C_{\mathcal{P}}\right\} \mid \Delta \mathcal{P}\right\rangle}{\left\langle\Re e\left\{C_{\mathcal{N}}\right\} \mid \Delta \mathcal{N}\right\rangle+\left\langle\Re e\left\{C_{\mathcal{P}}\right\} \mid \Delta \mathcal{P}\right\rangle}
$$

taking into account the impact of the longitudinal distribution of the carrier and photon densities, and the gain compression. The imaginary part of $C_{\mathcal{P}}$, corresponding to a modification of the refractive index induced by the photon density modification, is generally negligible. The maximum detuning is:

$$
\max \left\{\omega_{i}-\omega_{s}\right\}=\eta_{b}\left|f_{R}\right| \sqrt{1+\alpha_{e f f}^{2}}
$$

Since $\alpha_{e f f}$ is not a material parameter but rather a structural parameter, it depends in fact on the condition of injection. Consequently, the exact locking range must be calculated self-consistently. Stability of the solutions inside the locking range can be discussed using the Hurwitz criterion on the small signal equations.

\section{E. Determination of the power spectral densities of noise}

The longitudinal mode $Z_{L}(z)$ depends on $\omega, \mathcal{P}$ and $\mathcal{N}$. Consequently $\mathcal{P}(t)$ cannot be directy related to $I(t)$ using the group velocity. The following expression, determined by Tromborg et al. [47], is used for $\mathcal{P}(t)$ to take into account these dependences:

$$
\begin{aligned}
|\delta \mathcal{P}\rangle= & \left(1-\mathfrak{M}_{\mathcal{P}}\right)^{-1}\left[\left|\mathcal{P}_{0}\right\rangle \frac{\delta I}{I_{0}}+\frac{\left|H_{I}\right\rangle}{2 I_{0}} \frac{d \delta I}{d t}+\left|H_{\phi}\right\rangle \frac{d \phi}{d t}\right] \\
& +\mathfrak{M}|\delta \mathcal{N}\rangle
\end{aligned}
$$

Expression for the different functions and operators are recalled in Appendix B. The rate equations of (34), (35) and (36) are linearized around the static point $\left(I_{0}, I_{i n 0}, \theta_{0}, \mathcal{N}_{0}(z)\right)$. $\left(\delta I, \delta I_{i n}, \delta \theta, \delta \mathcal{N}(z)\right)$ is the deviation to the 
static point induced by noise. Due to the unstationarity of the phase noise, $\delta \phi$ or $\delta \phi_{i n}$ cannot be defined. Using the following vectorial notations [62]:

$$
\begin{aligned}
\boldsymbol{X}(\Omega)= & {\left[\begin{array}{c}
\delta I(\Omega) \\
\phi(\Omega) \\
\delta \mathcal{N}(\Omega, z)
\end{array}\right] } \\
\boldsymbol{X}_{\text {in }}(\Omega)= & {\left[\begin{array}{c}
\delta I_{\text {in }}(\Omega) \\
\phi_{\text {in }}(\Omega) \\
\delta \mathcal{N}_{\text {in }}(\Omega, z)
\end{array}\right] } \\
\boldsymbol{F}(\Omega)= & {\left[\begin{array}{c}
F_{I}(\Omega) \\
F_{\phi}(\Omega) \\
F_{\mathcal{N}}(\Omega, z)+\delta J(\Omega, z)
\end{array}\right] }
\end{aligned}
$$

the expression of the noises can be expressed by:

$$
\boldsymbol{X}(\Omega)=([M(\Omega)]+[D])^{-1}\left([C] \boldsymbol{X}_{\boldsymbol{i n}}(\Omega)+\boldsymbol{F}(\Omega)\right)
$$

Noting

$$
\begin{aligned}
& k_{c}=\eta_{b}\left|f_{R}\right| \cos \left(\theta_{0}+\psi_{R}\right) \\
& k_{s}=\eta_{b}\left|f_{R}\right| \sin \left(\theta_{0}+\psi_{R}\right)
\end{aligned}
$$

the coefficients of the matrix $[M],[D]$ and $[C]$ are given by:

$$
\begin{aligned}
m_{11}= & -2\left\langle C_{\mathcal{N}_{r}} \mid \Delta \mathcal{N}_{0}\right\rangle-2\left\langle C_{\mathcal{P} r} \mid \Delta \mathcal{P}_{0}\right\rangle \\
& -2\left\langle C_{\mathcal{P}_{r}}\left|\left(1-\mathfrak{M}_{\mathcal{P}}\right)^{-1}\right| \mathcal{P}_{0}\right\rangle \\
& -j \Omega\left\langle C_{\mathcal{P}_{r}}\left|\left(1-\mathfrak{M}_{\mathcal{P}}\right)^{-1}\right| H_{I}+j \Omega\right\rangle \\
m_{12}= & -j \Omega 2 I_{0}\left\langle C_{\mathcal{P} r}\left|\left(1-\mathfrak{M}_{\mathcal{P}}\right)^{-1}\right| H_{\phi}\right\rangle \\
m_{13}= & -2 I_{0}\left\langle C_{\mathcal{N} r}\right|-\left\langle C_{\mathcal{P} r}\right| \mathfrak{M} \\
m_{21}= & -\frac{1}{I_{0}}\left\langle C_{\mathcal{P}_{i}}\left|\left(1-\mathfrak{M}_{\mathcal{P}}\right)^{-1}\right| \mathcal{P}_{0}\right\rangle \\
& -\frac{j \Omega}{2 I_{0}}\left\langle C_{\mathcal{P} i}\left|\left(1-\mathfrak{M}_{\mathcal{P}}\right)^{-1}\right| H_{I}\right\rangle \\
m_{22}= & -j \Omega\left\langle C_{\mathcal{P}_{i}}\left|\left(1-\mathfrak{M}_{\mathcal{P}}\right)^{-1}\right| H_{\phi}+j \Omega\right\rangle \\
m_{23}= & -\left\langle C_{\mathcal{N}_{i}}\right|-\left\langle C_{\mathcal{P}_{i}}\right| \mathfrak{M}
\end{aligned}
$$




$$
\begin{aligned}
m_{31}= & {\left[v_{g} \frac{\partial g}{\partial \mathcal{P}} \mathcal{P}_{0}(z)+v_{g} g_{0}(z)\right] } \\
& \times\left(1-\mathfrak{M}_{\mathcal{P}}\right)^{-1}\left[\frac{\left|\mathcal{P}_{0}\right\rangle}{I_{0}}+\frac{j \Omega\left|H_{I}\right\rangle}{2 I_{0}}\right] \\
m_{32}= & {\left[v_{g} \frac{\partial g}{\partial \mathcal{P}} \mathcal{P}_{0}(z)+v_{g} g_{0}(z)\right] } \\
& \times\left(1-\mathfrak{M}_{\mathcal{P}}\right)^{-1}\left|H_{\phi}\right\rangle j \Omega \\
m_{33}= & \frac{\partial \mathrm{R}}{\partial \mathcal{N}}+v_{g} g_{N} \mathcal{P}_{0}(z) \\
& +\left[v_{g} \frac{\partial g}{\partial \mathcal{P}} \mathcal{P}_{0}(z)+v_{g} g_{0}(z)\right] \mathfrak{M}+j \Omega \\
d_{11}= & -k_{c} \\
d_{12}= & -2 I_{0} k_{s} \\
d_{21}= & -\frac{k_{s}}{2 I_{i n 0}} \\
d_{22}= & -k_{c} \\
c_{11}= & \frac{I_{0}}{I_{i n 0}} k_{c} \\
c_{12}= & -2 I_{0} k_{s} \\
c_{21}= & \frac{k_{s}}{2 I_{i n 0}} \\
c_{22}= & k_{c}
\end{aligned}
$$

The power spectral densities can be expressed using an Hermitian product:

$$
S_{\boldsymbol{X}}(\Omega) \delta\left(\Omega-\Omega^{\prime}\right)=\left\langle X(\Omega) X\left(\Omega^{\prime}\right)^{\dagger}\right\rangle
$$

The coefficients of $S_{\boldsymbol{X}}$ correspond to the power spectral densities of the intensity, phase and carrier density noises for the diagonal terms and to the respective interspectral power densities for the non diagonal ones. Using (48),

$$
\begin{aligned}
S_{\boldsymbol{X}}(\Omega)= & ([M]+[D])^{-1}[C] S_{\boldsymbol{X}_{i n}}(\Omega)[C]^{\dagger}([M]+[D])^{-1^{\dagger}} \\
& +([M]+[D])^{-1} S_{\boldsymbol{F}}(\Omega)([M]+[D])^{-1^{\dagger}}
\end{aligned}
$$

with

$$
S_{\boldsymbol{F}}(\Omega)=2\left[\begin{array}{ccc}
D_{I I} & 0 & D_{I \mathcal{N}}(z) \\
0 & D_{\phi \phi} & 0 \\
D_{I \mathcal{N}}(z) & 0 & D_{\mathcal{N N}}(z)+S_{J}(\Omega, z)
\end{array}\right]
$$

The linewidth of the injection locked spectrum can be determined from the value of $S_{\phi}(\Omega) / \Omega^{2}$ in $\Omega=0$. However, for very small values of $k_{c}, S_{\phi}(\Omega) / \Omega^{2}$ is not constant on a sufficiently wide range of frequencies around zero and consequently the power spectrum of the injection locked laser can no longer be represented by a Lorentzian lineshape. 


\section{F. Comments}

The coefficients which depend directly on the injected fields are $d_{i j}$ and $c_{i j} . c_{i j}$ indicates how the noise of the injected field $\boldsymbol{X}_{\boldsymbol{i n}}$ is coupled to the intrinsic sources of noise of the slave laser $\boldsymbol{F}$, resulting in an equivalent internal source of noise $[C] \boldsymbol{X}_{\boldsymbol{i n}}(\Omega)+\boldsymbol{F}(\Omega) . d_{i j}$ indicates how the injection modifies the response of the laser to the source of noise, which is represented by the coefficients $m_{i j}$. It is interesting to note that the coefficient $d_{i j}$ and $c_{i j}$ are independent on the structure, they are identical to the Fabry-Perot case [63]. Inversely, coefficients $m_{i j}$, which, without injection, determine the noise properties of the laser, depend on the structure of the laser. The terms involving a longitudinal integration take into account the effect of the spatial hole burning and non uniform current injection. The gain compression effect appears through $C_{\mathcal{P} r}$ and $\partial g / \partial \mathcal{P}$. The use of the expansion of the carrier density noise (44), involved new transfer of noise through the modification of the longitudinal mode represented by the operator $\mathfrak{M}_{\mathcal{P}}$ and $\mathfrak{M}$ and the functions $H(z)$ and $H_{I}(z)$. This effect leads to an additional coupling between the rate equations of intensity, phase and carrier density which appears specially in the factor $m_{12}$ and $m_{32}$, which would be null otherwise. Thus, factor $m_{12}$ corresponds to a contribution of the frequency noise to the intensity noise. Such a contribution is generally induced only by injection locking through the factor $d_{12}$ but is here also self-induced. It is due to the fact that the frequency noise induces a noise on the longitudinal distribution of the photon density and consequently a noise on the output power through the gain compression. Factor $m_{32}$ represents a contribution of the frequency noise on the carrier density noise, the frequency noise induces a noise on the longitudinal distribution of the photon density and consequently a noise on the carrier density through the photon-carrier coupling of the gain. Moreover the influence of those two parameters increase with the frequency $\Omega$ and consequently can become important for the calculation of the phase noise at frequency offset above $1 \mathrm{GHz}$. Finally, the gain curve influence is also present through the factor $H(z)$ which is important for emission far from the maximum of the gain curve.

\section{DYNAMIC EQUATIONS AND NOISE PROPERTIES OF LASER DIODE WITH FORWARD INJECTION}

We consider in this section the injection of a forward traveling wave $\vec{E}_{i n j L}^{+}$through the rear facet, i.e. forward injection.

\section{A. Temporal envelope of the electric field}

For injection through the rear facet, (11) becomes:

$$
E^{+}\left(l^{-}, \omega\right)=r_{L} r_{2} E^{+}\left(l^{-}, \omega\right)+t_{L} t_{12} E_{i n j L}^{+}\left(0^{-}, \omega\right)+F_{L}(\omega)
$$


Using the following first order expansion:

$$
\begin{aligned}
\left(\frac{t_{L}}{r_{L}}\right)(\omega, \mathcal{N}, \mathcal{P}) & =\left(\frac{t_{L}}{r_{L}}\right)\left(\omega_{s}, \mathcal{N}_{s}, \mathcal{P}_{s}\right) \\
& +\left(\frac{\partial \frac{t_{L}}{r_{L}}}{\partial \omega}\right)_{s}\left(\omega-\omega_{s}\right) \\
& +\int_{0}^{l}\left(\frac{\delta \frac{t_{L}}{r_{L}}}{\delta k}\right)_{s} \frac{\partial k}{\partial \mathcal{N}} \Delta \mathcal{N}(z) d z \\
& +\int_{0}^{l}\left(\frac{\delta \frac{t_{L}}{r_{L}}}{\delta k}\right)_{s} \frac{\partial k}{\partial \mathcal{P}} \Delta \mathcal{P}(z) d z
\end{aligned}
$$

the equation of motion of the temporal envelope of the forward traveling component of the intracavity electric field at the front facet is established by inverse Fourier transform:

$$
\begin{aligned}
\frac{d A^{+}}{d t}(t)= & -j\left(\omega_{s}-\omega_{i}\right) A^{+}(t) \\
& +A^{+}(t)\left[\left\langle C_{\mathcal{N}} \mid \Delta \mathcal{N}\right\rangle+\left\langle C_{\mathcal{P}} \mid \Delta \mathcal{P}\right\rangle\right] \\
& +f_{T} t_{12} A^{i}(t) \\
& +\left[\left\langle C_{\mathcal{N}}^{t} \mid \Delta \mathcal{N}\right\rangle+\left\langle C_{\mathcal{P}}^{t} \mid \Delta \mathcal{P}\right\rangle\right] t_{12} A^{i}(t) \\
& +\frac{f_{T}}{f_{t}} \frac{d A^{i}}{d t}(t)+j \frac{f_{T}}{f_{t}}\left(\omega_{i}-\omega_{s}\right) t_{12} A^{i}(t) \\
& +F_{A}(t)
\end{aligned}
$$

where

$$
\begin{aligned}
f_{T} & =f_{D} t_{L s} \\
C_{\mathcal{N}}^{t} & =f_{R}\left(\frac{\delta \frac{t_{L}}{r_{L}}}{\delta k} \frac{\partial k}{\partial \mathcal{N}}\right)_{s} \\
C_{\mathcal{P}}^{t} & =f_{R}\left(\frac{\delta \frac{t_{L}}{r_{L}}}{\delta k} \frac{\partial k}{\partial \mathcal{P}}\right)_{s} \\
\frac{r_{L}}{t_{L}} f_{t} & =j\left(\frac{\partial \frac{t_{L}}{\partial \omega}}{\partial \omega}\right)_{s}^{-1}
\end{aligned}
$$

The two first terms of the right hand side of (55) are identical to the case of backward injection, and the four following terms represents the contribution of the injected field. The feed-in rate $f_{T} t_{12}$ is different from the backward case. Four additional contributions from the injected field appear in the equation. Those contributions come from the effect of the single pass through the cavity by the injected field. The fourth terms represents the modification of the single pass gain and phase rotation due to the deviation of the carrier and photon densities. The fifth takes into account the delay induced by the single pass trip by introducing a contribution of the derivate of the envelope of the injected field, and the sixth comes from the detuning of the injected frequency from the natural frequency of the cavity. 


\section{B. Optical phase and power equations}

The equations of motion for the optical phase and the optical power are derived using the same method as for the backward case:

$$
\begin{aligned}
\frac{d I}{d t}(t)= & 2\left[\left\langle C_{\mathcal{N} r} \mid \Delta \mathcal{N}\right\rangle+\left\langle C_{\mathcal{P} r} \mid \Delta \mathcal{P}\right\rangle\right] I(t) \\
& +2\left|f_{T}\right| \sqrt{T_{1} T_{2} I_{i n}(t) I(t)} \cos \left(\theta(t)+\psi_{T}\right) \\
& +2\left|\left\langle C_{\mathcal{N}}^{t} \mid \Delta \mathcal{N}\right\rangle+\left\langle C_{\mathcal{P}}^{t} \mid \Delta \mathcal{P}\right\rangle\right| \sqrt{T_{1} T_{2} I_{i n}(t) I(t)} \\
& \times \cos \left(\theta(t)+\arg \left(\left\langle C_{\mathcal{N}}^{t} \mid \Delta \mathcal{N}\right\rangle+\left\langle C_{\mathcal{P}}^{t} \mid \Delta \mathcal{P}\right\rangle\right)\right) \\
& -2\left|\frac{f_{T}}{f_{t}}\right|\left(\omega_{i}-\omega_{s}\right) \sqrt{T_{1} T_{2} I_{i n}(t) I(t)} \sin \left(\theta(t)+\psi_{t}\right) \\
& +\left|\frac{f_{T}}{f_{t}}\right| \frac{d I_{i n}}{d t}(t) \sqrt{\frac{T_{1} T_{2} I_{i n}(t)}{I(t)}} \cos \left(\theta(t)+\psi_{t}\right) \\
& -2\left|\frac{f_{T}}{f_{t}}\right| \frac{d \phi_{i n}}{d t}(t) \sqrt{T_{1} T_{2} I_{i n}(t) I(t)} \sin \left(\theta(t)+\psi_{t}\right) \\
& +F_{I}(t)
\end{aligned}
$$

$$
\begin{aligned}
& \frac{d \phi}{d t}(t)=\left[\left\langle C_{\mathcal{N} i} \mid \Delta \mathcal{N}\right\rangle+\left\langle C_{\mathcal{P} i} \mid \Delta \mathcal{P}\right\rangle\right]-\left(\omega_{i}-\omega_{s}\right) \\
& +\left|f_{T}\right| \sqrt{\frac{T_{1} T_{2} I_{i n}(t)}{I(t)}} \sin \left(\theta(t)+\psi_{T}\right) \\
& +\left|\left\langle C_{\mathcal{N}}^{t} \mid \Delta \mathcal{N}\right\rangle+\left\langle C_{\mathcal{P}}^{t} \mid \Delta \mathcal{P}\right\rangle\right| \sqrt{T_{1} T_{2}} \sqrt{\frac{I_{i n}(t)}{I(t)}} \\
& \times \sin \left(\theta(t)+\arg \left(\left\langle C_{\mathcal{N}}^{t} \mid \Delta \mathcal{N}\right\rangle+\left\langle C_{\mathcal{P}}^{t} \mid \Delta \mathcal{P}\right\rangle\right)\right) \\
& +\left|\frac{f_{T}}{f_{t}}\right|\left(\omega_{i}-\omega_{s}\right) \sqrt{\frac{T_{1} T_{2} I_{i n}(t)}{I(t)}} \cos \left(\theta(t)+\psi_{t}\right) \\
& +\frac{1}{2}\left|\frac{f_{T}}{f_{t}}\right| \frac{d I_{i n}}{d t}(t) \sqrt{\frac{T_{1} T_{2}}{I_{i n}(t) I(t)}} \sin \left(\theta(t)+\psi_{t}\right) \\
& +\left|\frac{f_{T}}{f_{t}}\right| \frac{d \phi_{i n}}{d t}(t) \sqrt{\frac{T_{1} T_{2} I_{i n}(t)}{I(t)}} \cos \left(\theta(t)+\psi_{t}\right) \\
& +F_{\phi}(t)
\end{aligned}
$$

where $\psi_{t}=\arg \left\{f_{T} / F_{T}\right\}$. Due to the involvement of the derivative of the temporal envelope of the injected wave, corresponding contributions appear in the equations of motion of the phase and the power. 


\section{Calculation of the locking condition}

Locked states are solution of equations (36), (57) and (58) with all noise and derivative terms set to zero. When locking is achieved, phase difference and angular frequency detuning are related by:

$$
\begin{aligned}
& \omega_{i}-\omega_{s}= \\
& \frac{\eta_{f} \sqrt{1+\alpha_{e f f}^{2}}}{1-\eta_{f} \sqrt{1+\alpha_{e f f}^{2}}\left|\frac{f_{T}}{f_{t}}\right| \cos \left(\theta_{0}+\psi_{t}-\tan ^{-1}\left(\alpha_{e f f}\right)\right)} \\
& \times\left\{\left|f_{T}\right| \sin \left(\theta_{0}+\psi_{T}-\tan ^{-1}\left(\alpha_{e f f}\right)\right)\right. \\
& \left.\quad+\left|f_{\Delta}\right| \sin \left(\theta_{0}+\arg \left(f_{\Delta}\right)-\tan ^{-1}\left(\alpha_{e f f}\right)\right)\right\}
\end{aligned}
$$

where:

$$
f_{\Delta}=\left\langle C_{\mathcal{N}}^{t} \mid \Delta \mathcal{N}\right\rangle+\left\langle C_{\mathcal{P}}^{t} \mid \Delta \mathcal{P}\right\rangle
$$

and $\eta_{f}=\sqrt{\frac{I_{i n o} T_{1}}{I_{0} / T_{2}}}, \eta_{f}^{2}$ is the ratio between the optical power injected through the rear facet and the forward traveling internal optical power at the front facet. Because $f_{\Delta}$ depends on $\mathcal{P}$ and $\mathcal{N}$, the exact expression of the locking range has to be determined self-consistently.

It could appear surprising to obtain a different expression of the locking range for the backward and the forward injections. In the case of a symmetric laser $\left(T_{1}=T_{2}\right)$, if we consider the light emitted by the rear facet, the existence of two different expressions seems to allow solutions where for example the forward traveling components of the intracavity field is locked but not the backward one. This is in fact not the case, because the injection modifies the longitudinal distribution of the optical power inside the cavity, and leads to an asymmetry of the emitted power, even for symmetric laser, as was shown in [29] for a Fabry-Perot cavity. Consequently it is consistent to obtain different expressions for the forward and backward injections.

\section{Power spectral densities of noise}

Using the notations:

$$
\begin{aligned}
k_{c} & =\eta_{f}\left|f_{T}\right| \cos \left(\theta_{0}+\psi_{T}\right) \\
k_{s} & =\eta_{f}\left|f_{T}\right| \sin \left(\theta_{0}+\psi_{T}\right) \\
\chi_{t} & =\left|\frac{f_{T}}{f_{t}}\right| \\
\chi_{c} & =\chi_{t} \cos \left(\theta_{0}+\psi_{t}\right) \\
\chi_{s} & =\chi_{t} \sin \left(\theta_{0}+\psi_{t}\right) \\
\xi_{\mathcal{N} c} & =\eta\left|C_{\mathcal{N}}^{t}\right| \cos \left(\arg \left\{C_{\mathcal{N}}^{t}\right\}+\theta_{0}\right) \\
\xi_{\mathcal{N} s} & =\eta\left|C_{\mathcal{N}}^{t}\right| \sin \left(\arg \left\{C_{\mathcal{N}}^{t}\right\}+\theta_{0}\right) \\
\xi_{\mathcal{P} c} & =\eta\left|C_{\mathcal{P}}^{t}\right| \cos \left(\arg \left\{C_{\mathcal{P}}^{t}\right\}+\theta_{0}\right) \\
\xi_{\mathcal{P} s} & =\eta\left|C_{\mathcal{P}}^{t}\right| \sin \left(\arg \left\{C_{\mathcal{P}}^{t}\right\}+\theta_{0}\right)
\end{aligned}
$$


with the same method than for the backward case, the power spectral densities of the intensity, phase and carrier density noises are given by the following $c_{i j}$ and $d_{i j}$ coefficients and 48 :

$$
\begin{aligned}
& d_{11}=-k_{c}-\left[\left\langle\xi_{\mathcal{N} c} \mid \Delta \mathcal{N}_{0}\right\rangle+\left\langle\xi_{\mathcal{P} c} \mid \Delta \mathcal{P}_{0}\right\rangle\right] \\
& -\left\langle\xi_{\mathcal{P}_{c}}\left|\left(1-\mathfrak{M}_{\mathcal{P}}\right)^{-1}\right| 2 \mathcal{P}_{0}\right\rangle \\
& -j \Omega\left\langle\xi_{\mathcal{P}_{c}}\left|\left(1-\mathfrak{M}_{\mathcal{P}}\right)^{-1}\right| H_{I}\right\rangle \\
& +\chi_{s}\left(\omega_{i}-\omega_{s}\right) \\
& d_{12}=-2 I_{0}\left\{k_{s}+\left[\left\langle\xi_{\mathcal{N} s} \mid \Delta \mathcal{N}_{0}\right\rangle+\left\langle\xi_{\mathcal{P} s} \mid \Delta \mathcal{P}_{0}\right\rangle\right]\right. \\
& \left.+j \Omega\left\langle\xi_{\mathcal{P} c}\left|\left(1-\mathfrak{M}_{\mathcal{P}}\right)^{-1}\right| H_{\phi}\right\rangle+\chi_{c}\left(\omega_{i}-\omega_{s}\right)\right\} \\
& d_{13}=2 I_{0}\left\langle\xi_{\mathcal{N} c}\right|+2 I_{0}\left\langle\xi_{\mathcal{P}_{c}}\right| \mathfrak{M} \\
& d_{21}=\frac{1}{2 I_{0}}\left\{k_{s}+\left[\left\langle\xi_{\mathcal{N} s} \mid \Delta \mathcal{N}_{0}\right\rangle+\left\langle\xi_{\mathcal{S} s} \mid \Delta \mathcal{P}_{0}\right\rangle\right]\right. \\
& -\left\langle\xi_{\mathcal{P} s}\left|\left(1-\mathfrak{M}_{\mathcal{P}}\right)^{-1}\right| \mathcal{P}_{0}\right\rangle \\
& \left.-\frac{j \Omega}{2}\left\langle\xi_{\mathcal{P}_{s}}\left|\left(1-\mathfrak{M}_{\mathcal{P}}\right)^{-1}\right| H_{I}\right\rangle+\chi_{c}\left(\omega_{i}-\omega_{s}\right)\right\} \\
& d_{22}=k_{c}+\left\langle\xi_{\mathcal{N} c} \mid \Delta \mathcal{N}_{0}\right\rangle+\left\langle\xi_{\mathcal{P}_{c}} \mid \Delta \mathcal{P}_{0}\right\rangle \\
& +j \Omega\left\langle\xi_{\mathcal{P} s}\left|\left(1-\mathfrak{M}_{\mathcal{P}}\right)^{-1}\right| H_{\phi}\right\rangle-\chi_{s}\left(\omega_{i}-\omega_{s}\right) \\
& d_{23}=\left\langle\xi_{\mathcal{N} s}\right|+\left\langle\xi_{\mathcal{P} s}\right| \mathfrak{M} \\
& c_{11}=\frac{I_{0}}{I_{\text {in } 0}}\left\{k_{c}+\left[\left\langle\xi_{\mathcal{N}_{c}} \mid \Delta \mathcal{N}_{0}\right\rangle+\left\langle\xi_{\mathcal{P} c} \mid \Delta \mathcal{P}_{0}\right\rangle\right]\right. \\
& \left.-\chi_{s}\left(\omega_{i}-\omega_{s}\right)\right\}+j \Omega \chi_{c} \\
& c_{12}=-2 I_{0}\left\{k_{s}+\left[\left\langle\xi_{\mathcal{N} s} \mid \Delta \mathcal{N}_{0}\right\rangle+\left\langle\xi_{\mathcal{P} s} \mid \Delta \mathcal{P}_{0}\right\rangle\right]\right. \\
& \left.+\chi_{c}\left(\omega_{i}-\omega_{s}\right)+j \Omega \chi_{s}\right\} \\
& c_{21}=\frac{1}{2 I_{\text {in } 0}}\left\{k_{s}+\left[\left\langle\xi_{\mathcal{N} s} \mid \Delta \mathcal{N}_{0}\right\rangle+\left\langle\xi_{\mathcal{P}_{s}} \mid \Delta \mathcal{P}_{0}\right\rangle\right]\right. \\
& \left.+\chi_{c}\left(\omega_{i}-\omega_{s}\right)+j \Omega \chi_{s}\right\} \\
& c_{22}=k_{c}+\left[\left\langle\xi_{\mathcal{N} c} \mid \Delta \mathcal{N}_{0}\right\rangle+\left\langle\xi_{\mathcal{P}_{c}} \mid \Delta \mathcal{P}_{0}\right\rangle\right] \\
& -\chi_{s}\left(\omega_{i}-\omega_{s}\right)+j \Omega \chi_{c}
\end{aligned}
$$

\section{E. Comments}

In difference with the backward case, the coefficients $c_{i j}$ and $d_{i j}$, depend on the structure of the laser. The coefficients $m_{i j}$ remain identical to the backward case since they represent the noise properties of the laser without injection. It can be also noticed that all the coefficients $c_{i j}$ and $d_{i j}$, excepted $d_{13}$ and $d_{23}$, contain a component proportional to the frequency $\Omega$. This contribution is particularly important for the characterization of the phase 
and intensity noise spectrum at large frequencies. For frequency closed to $f_{D}$, the Taylor expansion (16) and (54) should be extended to higher orders in $\left(\omega-\omega_{s}\right)$ leading to higher order temporal derivates in (55) and polynomes in $\Omega$ instead of linear terms in the expression of $c_{i j}$ and $d_{i j}$.

\section{Application to Fabry-Perot and DFB lasers}

\section{A. Injection locking of a Fabry-Perot}

We discuss in this section the application of the equations previously derived to a Fabry-Perot cavity. The field is considered to be uniform along the cavity, the emission to be near the maximum of the gain curve and the gain compression to be negligible. These hypothesis are fulfilled with a Fabry-Perot laser with facet reflectivities above 0.5 , operating not too far from the threshold [64].

For the case of backward injection:

$$
\begin{aligned}
f_{D} & =\frac{1}{\tau_{i n}}=\frac{v_{g}}{2 l} \\
C_{\mathcal{N}} & =\frac{1+j \alpha_{H}}{2 l} v_{g} \frac{\partial g}{\partial \mathcal{N}} \\
r_{L s} & =\frac{1}{r_{2}} \\
f_{R} & =\frac{v_{g}}{2 l r_{2}}
\end{aligned}
$$

Consequently, from (19), the equation of motion of $A^{+}$is:

$$
\begin{aligned}
\frac{d A^{+}}{d t}(t)= & -j\left(\omega_{s}-\omega_{i}\right) A^{+}(t) \\
& +\frac{1+j \alpha_{H}}{2} v_{g} \frac{\partial g}{\partial \mathcal{N}} \Delta \mathcal{N}(t) A^{+}(t) \\
& +\frac{v_{g}}{2 l} \frac{t_{32}}{r_{2}} A^{i}(t)+F_{A}(t)
\end{aligned}
$$

This equation is similar to the well known equation used by R. Lang [16]. However, the feed-in rate used by Lang is $c /(2 n l)$ whereas in our case the feed-in rate is $c /\left(2 l n_{g} r_{2}\right)$. We can notice two differences, the use of the group index and the involvement of the facet reflectivity $r_{2}$. The phenomenological argument of Lang was that the injected field adds $t_{32} A_{i}$ every time it hits the irradiated facet at a time interval equal to the cavity round trip time. However, the injected field is added only to the backward traveling wave reflected by the front facet with a coefficient of reflection $r_{2}$. Consequently when the forward traveling wave is considered, the feed-in rate is equal to the inverse of the round trip time divided by $r_{2}$. In many papers on injection locking, the $1 / r_{2}$ is missing. This problem is analog to the difference which has been noticed [65] between Kurokawa's [66] and Adler's [67] locking bandwidth due to the existence of different $Q$ factors, the resonator unloaded $Q$, the loaded $Q$ and the external $Q$. The expression of the feed-in rate derived in [15] using the round trip time traveling wave amplifier model is equal to our expression. The expression of the feed-in rate given in [20] seems also to agree with our expression, however it is not clear whether it was established for the total intracavity electric field or for the forward traveling component. For the envelope of the total intracavity electric field at the front facet, the feed-in rate is $c\left(1+r_{2}\right) /\left(2 \ln _{g} r_{2}\right)$, it 
is in agreement with the value obtained in [28] which uses for the mirror the model of a dielectric plan where a surface current is induced by the electric field instead of the boundary conditions used in this article.

In the forward case:

$$
\begin{aligned}
t_{L s} & =\frac{1}{\sqrt{r_{1} r_{2}}} \\
f_{T} & =\frac{1}{\tau_{i n} \sqrt{r_{1} r_{2}}} \\
f_{t} & =\frac{2}{\tau_{i n}} \\
C_{\mathcal{N}}^{t} & =-\frac{1+j \alpha_{H}}{4 l \sqrt{r_{1} r_{2}}} \frac{\partial g}{\partial \mathcal{N}} v_{g}
\end{aligned}
$$

and (55) becomes:

$$
\begin{aligned}
\frac{d A^{+}}{d t}(t)= & j\left(\omega_{s}-\omega_{i}\right) A^{+}(t) \\
& +\frac{1+j \alpha_{H}}{2} v_{g} \frac{\partial g}{\partial \mathcal{N}} \Delta \mathcal{N} A^{+}(t) \\
& +\frac{t_{12}}{\tau_{i n} \sqrt{r_{1} r_{2}}} A^{i}(t) \\
& -\frac{\left(1+j \alpha_{H}\right)}{4 \sqrt{r_{1} r_{2}}} \frac{\partial g}{\partial \mathcal{N}} v_{g} \Delta \mathcal{N} A^{i}(t) \\
& -j \frac{t_{12}}{2 \sqrt{r_{1} r_{2}}}\left(\omega_{s}-\omega_{i}\right) A^{i}(t) \\
& +\frac{t_{12}}{2 \sqrt{r_{1} r_{2}}} \frac{d A^{i}}{d t}(t)+F_{A}(t)
\end{aligned}
$$

As previously noticed, the total feed-in rate can be decomposed in three terms, $\kappa_{i n}=\kappa_{i n 1}+\kappa_{\text {in } 2}+\kappa_{i n 3}$ :

$$
\begin{aligned}
\kappa_{i n 1} & =\frac{1}{\tau_{i n} \sqrt{r_{1} r_{2}}} \\
\kappa_{i n 2} & =-\frac{\left(1+j \alpha_{H}\right)}{4 \sqrt{r_{1} r_{2}}} \frac{\partial g}{\partial \mathcal{N}} v_{g} \Delta \mathcal{N} \\
\kappa_{i n 3} & =-j \frac{\omega_{s}-\omega_{i}}{2 \sqrt{r_{1} r_{2}}}
\end{aligned}
$$

$\kappa_{i n 1}$ is the main term of $\kappa_{i n}$. It is identical to the backward case only for a symmetric laser. The others terms of $\kappa_{i n}$ can become noticeable when the cavity is particularly long or when locking is achieved with very large detuning. Finally, the contribution of the derivative of the envelope of the injected electric field can become noticeable when the variation of $A^{i}$ during a round trip time is non negligible, for example when the master laser is modulated at high frequency which is an important point in telecommunication applications.

\section{B. Numerical applications for DFB laser with an anti-reflexion coating}

We will now present numerical results for the parameters $f_{R}, f_{T} \chi_{t}$ for DFB lasers with an anti-reflection coated front facet. 
1) Parameters of injection of a DFB laser with an anti-reflection coated facet: For the Fabry-Perot cavity, $f_{D}$ represents the inverse of the round-trip time of the wave between the two reflective facets. However, in a DFB laser, the definition of the round-trip time is not straightforward due to the distributed reflexion added to the facet reflexions. Consequently $1 / f_{D}$ represents an effective round-trip time for a laser containing distributed reflexions. The resolution of the coupled equations for a DFB laser [56] with coupling coefficient of the grating $\kappa$, Bragg wave number $\beta_{B}$ and grating phase $\Omega_{B}$ gives:

$$
r_{L}=\frac{\left(\kappa+\delta \rho_{1}\right) \tanh (\gamma l)+j \gamma \rho_{1}}{-\left(\kappa \rho_{1}+\delta\right) \tanh (\gamma l)+j \gamma} e^{-j \Omega}
$$

with $\delta=k-\beta_{B}$, the complex detuning from the Bragg wave number, $\rho_{1}=r_{1} e^{j\left(\Omega_{B}-2 \beta_{B} l\right)}$ and $\gamma=\sqrt{\kappa^{2}-\delta^{2}}$, the complex wave number of the longitudinal envelope. For an anti-reflection coated front facet laser, the resonnance condition is $1 / r_{L s}=0$, consequently:

$$
\tanh (\gamma l)=\frac{j \gamma}{\kappa \rho_{1}+\delta}
$$

The expression of $f_{R}$ is calculated from (85) and (86):

$$
f_{R}=-j \frac{v_{g}}{l} \frac{\left[(\kappa l)^{2}-(\gamma l)^{2}\right]\left[\left(1+\rho_{1}^{2}\right)+2 \rho_{1} \frac{\gamma}{\kappa}\right]}{(\gamma l)\left[\rho_{1}+j(\kappa l)\left(1+\rho_{1}^{2}\right)\right]+\kappa l+2 j \rho_{1}(\gamma l)^{2}}
$$

For forward injection, the additional parameter $f_{t}$ is derived from $t_{L} / r_{L}$, calculated from the solution of the coupled equations:

$$
\frac{t_{L}}{r_{L}}=\frac{\gamma e^{j \beta_{B} l}}{-j \kappa^{*} e^{-j \Omega} \sinh (\gamma l)+r_{1}[\cosh (\gamma l)-j \delta \sinh (\gamma l)]}
$$

We will now present numerical results for the parameter of injection in forward and backward configuration.

Fig. 2. Normalised feed-in rate for the backward injection (left) and forward injection (right)

2) Case of a real rear reflectivity: Fig. 2(left) shows the modulus of the feed-in rate $f_{R}$ as a function of the normalized coupling coefficient of the grating $\kappa . l$ for four different values of the rear facet reflectivity. The feed-in rate is normalized by the free spectral range of a Fabry-Perot laser of the same length. The normalized values of the feed-in rate are distributed around two curves. The higher curve corresponds to a wavelength lower than the Bragg wavelength, whereas the lower curve corresponds to a higher wavelength. The discontinuity for $r_{1}=0.565$, $\kappa . l=1.75$ and $r_{1}=0.775, \kappa . l=3.25$ are due to a mode hopping corresponding to a change of the sign of the detuning from the Bragg frequency. The normalized value of the feed-in rate for a cleaved facet Fabry-Perot laser ( $r=-0.565$ ) is 1.8. Consequently, for normalized coupling coefficients of the grating lower than 1.5, the feed-in rate of the DFB laser is higher than for a Fabry-Perot laser, in the limit of a rear facet reflectivity lower than 0.95. For higher coupling coefficient of the grating and intermediate values of the rear facet reflectivity, the feed-in rate of the DFB is lower than for a Fabry-Perot laser. For strong coupling the influence of the boundary conditions is weaker due to the predominance of the distributed reflexion, and consequently the feed-in rate is weaker. Moreover the feed-in rate tends toward zero for very high coupling coefficients regardless of the rear reflectivity.In contrast, 
for weak coupling, the value of the rear reflectivity and the position of the lasing wavelength have an important influence on the feed-in rate.

On Fig. 2(right) are shown the normalized modulus of the primary feed-in rate $\left(f_{T}\right)$ for forward injection as a function of the normalized coupling coefficient to the grating and for four different values of the reflectivity of the rear facet. For a cleaved facet Fabry-Perot laser, the normalized feed-in rate is equal to 1.8. Consequently, the primary feed-in rate of the DFB laser becomes lower than for a Fabry-Perot laser for coupling coefficients higher than 1.7. The effect of the mode hopping for $r_{1}=0.775$ and 0.565 is indicated by the two arrows and is weaker than for the backward case.

Fig. 3. $\chi_{t}$ as a function of the coupling coefficient of the grating

Fig. 4. Argument of the feed in rate for the backward and forward injection (left) and phase shift between $f_{T}$ and $f_{t}$ (right)

On Fig. 3 are represented the parameter $\chi_{t}$ specific to the forward injection as a function of the normalized coefficient of the grating and for four different rear reflectivities. We notice than the values do not exhibit the same decay curve as in the case of $f_{R}$ and $f_{T}$. For a wavelength lower than the Bragg wavelength $\left(r_{1}=0.95, r_{1}=0.775\right.$ and $\kappa . l>1.7, r_{1}=0.565$ and $\left.\kappa . l>3.2\right), \chi_{t}$ increases with $\kappa . l$ and for a wavelength higher $\left(r_{1}=0.25, r_{1}=0.775\right.$ and $\kappa . l<1.7, r_{1}=0.565$ and $\left.\kappa . l<3.2\right)$ it decreases. The value of $\chi_{t}$ for a cleaved facet Fabry-Perot is 0.9 , consequently the additional coupling measured by $\chi_{t}$ is weaker in a DFB laser than in a Fabry-Perot laser.

Finally, Fig. 4 presents the arguments of $f_{R}, f_{T}$ and $f_{T} / f_{t}$ in degrees as a function of the normalized coupling coefficient of the grating for three different values of the rear reflectivity. The change of position of the mode indicated by the arrow is associated to a phase rotation of nearly $180^{\circ}$ of $f_{R}$ and $90^{\circ}$ of $f_{T}$. Arguments of $f_{T} / f_{t}$ exhibits a behavior similar to the argument of $f_{R}$.

3) Lasing mode in the forbidden band: We have previously studied the case of a DFB laser with an anti-reflection coated front facet and a rear facet with a real reflectivity. The case of a purely imaginary rear reflectivity is also interesting because in this case the principal mode of the laser is in the center of the forbidden band [17].

Fig. 5. Parameters as a function of the reflectivity for two different coefficients (a) threshold gain, (b) forward feed-in rate, (c) backward feed-in rate, (d) $\chi_{t}$ forward parameter

In Fig. 5 are shown (a) the normalized threshold gain $\alpha_{0}$, (b) (c) the normalized modulus of the feed-in rates $f_{R}, f_{T}$, (d) the parameter $\chi_{t}$, as a function of the modulus of the rear reflectivity. Only the reflectivities for which the Bragg mode has the lowest threshold have been considered. Two coupling coefficients of the grating have been used, $\kappa . l=1$ and $\kappa . l=0.7$. The value of $f_{R}$ strongly varies with the reflectivity and becomes extremely high for $r_{1}=j 0.487(\kappa . l=1)$ and $r_{1}=j 0.62(\kappa . l=0.7)$. By comparison, Fig. 2(left) presents, for real reflectivities, ranges of normalized value of the module of the feed-in rate of $[4.5,9.5]$ for $\kappa . l=0.7$, and $[2.7,7]$ for $\kappa . l=1$. For 
high values of $r_{1}$, the feed-in rate is practically two orders of magnitude below the feed-in rate of the cleaved facet Fabry-Perot laser. These results are important since they demonstrate that in choosing appropriately the value of the rear reflectivity, the feed-in rate, and consequently the locking range, can become extremely high or extremely small.

We do not find the same large range of values for the primary feed-in rate $f_{R}$ in the forward injection. However Fig. 5 shows that it increases with the reflectivity which is inverse to the case of a real reflectivity. Moreover, the sensitivity to the coupling coefficient of the grating is higher.

Finally, the results presented for the parameter $\chi_{t}$ exhibit, as for the real reflectivity case, values lower than for the cleaved facet Fabry-Perot laser. It is interesting to note that for every value of $\kappa . l$, there is a value of $r_{1}$ for which $\chi_{t}$ is zero.

4) Influence of the phase of the coefficient of reflection: We have shown that results for real and purely imaginary reflectivity are strongly different. Consequently we will now study the impact of the argument of the rear reflectivity on the different parameters considered.

Fig. 6. Influence of the phase of the reflectivity on the parameter of injection (a) threshold gain and Bragg detuning, (b) forward feed-in rate, (c) backward feed-in rate, (d) $\chi_{t}$ forward parameter

In Fig. 6 are shown the normalized Bragg detuning and threshold gain (a), the modulus of the normalized feed-in rate $f_{R}$ for backward injection (b), the modulus of the normalized primary feed-in rate $f_{T}(\mathrm{c})$ and the parameter $\chi_{t}$ for forward injection (d), all as a function of the argument of the rear reflectivity. The results concerning the feed-in rate $f_{R}$ show that it has a quadratic behavior centered around the case of the Bragg mode $(\pi / 2)$. Consequently the influence of the argument is particularly important near the real axis. For the forward injection, the Bragg mode corresponds to a local maximum of $f_{T}$. A phase shift of $45^{\circ}$ from the imaginary axis induces a decrease of the primary feed-in rate of $30 \%$. Between $45^{\circ}$ and $-90^{\circ}$ and between $135^{\circ}$ and $270^{\circ}, f_{T}$ depends quasi linearly on the argument of the reflectivity. Finally, the Bragg mode corresponds to a minimum for the parameter $\chi_{t}$. In opposition with $f_{R}$, the Bragg mode is the case where the influence of the argument of the reflectivity is the highest. Once again, all the values of $\chi_{t}$ presented are below the case of the cleaved facet Fabry-Perot cavity.

\section{CONCLUSION}

We have presented a theory for the injection locking of multisection lasers taking into account spatial hole burning, the non linear gain and the direction of injection. The contribution of the spontaneous emission has been treated with the Green's function method and the contribution of the injected fields using effective reflection and transmission coefficients accounting for longitudinal distribution of the carrier and photon densities. Two different equations of evolution for the complex envelope of the forward component of the intracavity electric field at the front facet have been derived, corresponding to the two possible directions of injection. This analysis shows that backward injection corresponds to the classical equation of R. Lang, whereas forward injection leads to additional 
contributions. Part of those additional contributions has been previously found in the equation derived by Tromborg et al. [31], but the contribution of the detuning was missing and it was not pointed out that those contributions were related to the facet of injection. The paper provides analytical expressions of the noise power spectral densities including mathematical tools presented in [47]. Those expressions are interesting for accurate characterization of the phase noise spectrum of injection locked laser, especially for forward injection and for injection of modulated light. Moreover, they show that injection locked lasers can exhibit asymmetric noise properties, which has been experimentally demonstrated in laser submitted to external feedback [68]. The application of our general equation to Fabry-Perot laser shows that the expression of the feed-in rate considered in the literature is sometimes incomplete. Numerical results show that DFB lasers can be designwith very large feed-in rate for backward injection, or with large feed-in rate for forward injection and very small one for backward injection.

\section{APPENDIX A}

\section{RESOLUTION OF THE INHOMOGENEOUS HELMOTZ EQUATION WITH GREEN'S FUNCTION}

Knowing two independent solutions $Z_{1}$ et $Z_{2}$ of the homogeneous Helmotz equation, i.e. two solutions with Wronskian

$$
W=Z_{1} d Z_{2} / d z-Z_{2} d Z_{1} / d z
$$

different from zero, the general solution of the inhomogeneous equation can be expressed as:

$$
\begin{aligned}
E(z, \omega)= & Z_{0}(z) \\
& +\int_{z}^{l} f\left(z, \omega^{\prime}\right) \frac{\left[Z_{1}(z) Z_{2}\left(z^{\prime}\right)-Z_{2}(z) Z_{1}\left(z^{\prime}\right)\right]}{W} d z^{\prime}
\end{aligned}
$$

with $Z_{0}$ is a solution of the homogeneous equation determined by the boundary conditions:

$$
\begin{aligned}
& E^{+}\left(0^{+}, \omega\right)=r_{1} E^{-}\left(0^{+}, \omega\right)+t_{12} E_{i n j L}^{+}\left(0^{-}, \omega\right) \\
& E^{-}\left(l^{-}, \omega\right)=r_{2} E^{+}\left(l^{-}, \omega\right)+t_{32} E_{i n j R}^{-}\left(l^{+}, \omega\right)
\end{aligned}
$$

$E_{i n j L}^{+}\left(0^{-}, \omega\right)$ and $E_{i n j R}^{-}\left(l^{+}, \omega\right)$ are the projection of the external field on the transverse mode and polarization vector of the cavity in, respectively, $0^{-}$and $l^{+}$:

$$
E_{i n j}(z, \omega)=\frac{\iint \vec{E}_{e x t}(x, y, z, \omega) \Phi(x, y) \vec{u} d x d y}{\iint \Phi(x, y) \Phi(x, y) d x d y}
$$

We choose $Z_{2}$ satisfying the left boundary condition without injection. Consequently, the left boundary condition gives:

$$
\begin{aligned}
Z_{0}^{+}(0)-r_{1} Z_{0}^{-}(0)= & \left(r_{1} Z_{1}^{-}(0)-Z_{1}^{+}(0)\right) \int_{0}^{l} f\left(\omega, z^{\prime}\right) \frac{Z_{2}\left(z^{\prime}\right)}{W} d z^{\prime} \\
& +t_{12} E_{i n j L}^{+}\left(0^{-}\right)
\end{aligned}
$$


If $Z_{1}$ satisfies $Z_{1}(0)=0$ and $d Z_{1}(0) / d z=1$ such that $W=\left(1+r_{1}\right) Z_{2}^{-}(0)$, since $Z_{0}$ is a solution of the homogeneous equation, $Z_{2}$ satisfies the left boundary condition and $E\left(l^{-}, \omega\right)=Z_{0}(l)$ :

$$
\begin{aligned}
Z_{0}^{+}(l) Z_{2}^{+}(l)-Z_{0}^{-}(l) Z_{2}^{-}(l)= & Z_{1}^{+}(0) \int_{0}^{l} f\left(\omega, z^{\prime}\right) \frac{Z_{2}\left(z^{\prime}\right)}{W} d z^{\prime} \\
& +Z_{2}^{-}(0) t_{12} E_{i n j L}^{+}\left(0^{-}\right)
\end{aligned}
$$

Using now the right boundary condition and defining the reflectivity $r_{L}$ and the transmission coefficient $t_{L}$ :

$$
\begin{aligned}
r_{L} & =Z_{L}^{+}\left(l^{-}\right) / Z_{L}^{-}\left(l^{-}\right) \\
t_{L} & =Z_{L}^{+}\left(0^{+}\right) / Z_{L}^{-}\left(l^{-}\right) \\
F_{L}(\omega) & =\frac{Z_{1}^{+}(0)}{Z_{L}^{-}(l)} \int_{0}^{l} f\left(z^{\prime}, \omega\right) Z_{L}\left(z^{\prime}\right) d z^{\prime}
\end{aligned}
$$

we obtain the following equation for $E^{+}\left(l^{-}, \omega\right)$

$$
\begin{aligned}
E^{+}\left(l^{-}, \omega\right)= & r_{2} r_{L} E^{+}\left(l^{-}, \omega\right)+t_{L} t_{12} E_{i n j L}^{+}\left(0^{-}, \omega\right) \\
& +r_{L} t_{32} E_{i n j R}^{-}\left(l^{+}, \omega\right)+F_{L}(\omega)
\end{aligned}
$$

\section{APPENDIX B}

\section{EXPRESSION OF THE PHOTON LONGITUDINAL DENSITY}

Equation (44) is the expansion of the photon density established by Tromborg et al. [47]. In this appendix, we briefly remind the method and the expression of the main parameters. The longitudinal mode is expended at first order in $\omega, \mathcal{P}$ and $\mathcal{N}$ :

$$
Z_{L}(z)=Z_{L 0}(z)+\frac{\partial Z_{L}}{\partial \omega}\left(\omega-\omega_{s}\right)+\left\langle Z_{\mathcal{N}}(z) \mid \delta \mathcal{N}\right\rangle+\left\langle Z_{\mathcal{P}}(z) \mid \delta \mathcal{P}\right\rangle
$$

with

$$
\begin{aligned}
& \frac{\delta Z_{L}(z)}{\delta k\left(z^{\prime}\right)} \frac{\partial k_{0}}{\partial \mathcal{N}}=Z_{\mathcal{N}}\left(z, z^{\prime}\right) \\
& \frac{\delta Z_{L}(z)}{\delta k\left(z^{\prime}\right)} \frac{\partial k_{0}}{\partial \mathcal{P}}=Z_{\mathcal{P}}\left(z, z^{\prime}\right)
\end{aligned}
$$

$Z_{L 0}(z)$ is the stationary longitudinal mode with injection. Using (13) and Fourier transform, we obtain:

$$
\begin{aligned}
|\delta \mathcal{P}\rangle= & \mathfrak{M}_{\mathcal{N}}|\delta \mathcal{N}\rangle+\mathfrak{M}_{\mathcal{P}}|\delta \mathcal{P}\rangle \\
& +\left[\left|\mathcal{P}_{0}\right\rangle \frac{\delta I}{I_{0}}+\frac{\left|H_{I}\right\rangle}{2 I_{0}} \frac{d \delta I}{d t}+\left|H_{\phi}\right\rangle \frac{d \phi}{d t}\right]
\end{aligned}
$$

where two operators are defined:

$$
\begin{aligned}
& \mathfrak{M}_{\mathcal{P}}=\frac{2 \Re e\left\{Z_{L 0}(z)\right\}}{\left|Z_{L O}(z)\right|^{2}}\left|\mathcal{P}_{0}\right\rangle\left\langle Z_{\mathcal{P}}(z)\right| \\
& \mathfrak{M}_{\mathcal{N}}=\frac{2 \Re e\left\{Z_{L 0}(z)\right\}}{\left|Z_{L O}(z)\right|^{2}}\left|S_{0}\right\rangle\left\langle Z_{\mathcal{N}}(z)\right|
\end{aligned}
$$


Finally, supposing $1-\mathfrak{M}_{\mathcal{P}}$ inversible and noting:

$$
\mathfrak{M}=\left(1-\mathfrak{M}_{\mathcal{P}}\right)^{-1} \mathfrak{M}_{\mathcal{N}}
$$

we obtain [47]:

$$
\begin{aligned}
|\delta P\rangle= & \left(1-\mathfrak{M}_{\mathcal{P}}\right)^{-1}\left[\left|\mathcal{P}_{0}\right\rangle \frac{\delta I}{I_{0}}+\frac{\left|H_{I}\right\rangle}{2 I_{0}} \frac{d \delta I}{d t}+\left|H_{\phi}\right\rangle \frac{d \phi}{d t}\right] \\
& +\mathfrak{M}|\delta \mathcal{N}\rangle
\end{aligned}
$$

$H_{\phi}(z)$ and $H_{I}(z)$ come from the $\omega$ dependance of $Z_{L}$ :

$$
\begin{aligned}
& H_{\phi}(z)=2 \Re e\left\{I_{0} Z_{L 0}^{*}(z) \frac{\partial Z_{L}}{\partial \omega}\left(z, \omega_{s}\right)\right\} \\
& H_{I}(z)=2 \Im m\left\{I_{0} Z_{L 0}^{*}(z) \frac{\partial Z_{L}}{\partial \omega}\left(z, \omega_{s}\right)\right\}
\end{aligned}
$$

\section{REFERENCES}

[1] S. Kobayashi, Y. Yamamoto, and T. Kimura, "Optical FM signal amplification and FM noise-reduction in an injection locked AlGaAs semiconductor laser," Electronics Letters, vol. 17, no. 22, pp. 849-851, 1981.

[2] K. Kikuchi, C.-E. Zah, and T. Lee, "Amplitude-modulation sideband injection locking characteristics of semiconductor lasers and their application," Journal of Lightwave Technology, vol. 6, no. 12, pp. 1821-1830, 1988.

[3] T. B. Simpson, J. M. Liu, and G. A., "Bandwidth enhancement and broadband noise reduction in injection-locked semiconductor lasers," IEEE Photonics Technology Letters, vol. 7, no. 7, pp. 709-711, 1995.

[4] L. A. Johansson and A. J. Seeds, "Millimeter-wave modulated optical signal generation with high spectral purity and wide-locking bandwidth using a fiber-integrated optical injection phase-lock loop," IEEE Photonics Technology Letters, vol. 12, no. 6, pp. 690-692, 2000.

[5] C. W. Chow, C. S. Wong, and H. K. Tsang, "All-optical modulation format conversion and multicasting using injection-locked laser diodes," Journal of Lightwave Technology, vol. 22, no. 11, pp. 2386-2392, 2004.

[6] H. Wang, M. J. Freeman, and D. G. Steel, "Squeezed light from injection-locked quantum well lasers," Physical Review Letters, vol. 71, no. 24, pp. 3951-3954, 1993.

[7] J. Jahanpanah and R. Loudon, "Theory of laser-amplifier injection locking," Physical Review A, vol. 54, no. 6, pp. 5210-5226, 1996.

[8] A. Murakami and J. Ohtsubo, "Synchronization of feedback-induced chaos in semiconductor lasers by optical injection," Physical Review A, vol. 65, no. 3, p. 033826, 2002.

[9] D. Goulding, S. P. Hegarty, O. Rasskazov, S. Melnil, M. Hartnett, G. Greene, J. G. McInerney, D. Rachinskii, and G. Huyet, "Excitability in a quantum dot semiconductor laser with optical injection," Physical Review Letters, vol. 98, no. 15, p. 153903, 2007.

[10] J. M. Liu and T. B. Simpson, "Characterization of fundamental parameters of a semiconductor laser with an injected optical probe," IEEE Photonics Technology Letters, vol. 5, no. 4, pp. 380-382, 1993.

[11] M. P. van Exter, C. Biever, and J. P. Woerdman, "Effect of optical injection on bias voltage and spectrum of a semiconductor laser," IEEE Journal of Quantum Electronics, vol. 29, no. 11, pp. 2771 -2779, 1993.

[12] H. L. Stover and W. Steier, "Locking of laser oscillators by light injection," Applied Physical Letters, vol. 8, pp. 91-93, 1966.

[13] S. Kobayashi and T. Kimura, "Injection locking in AlGaAs semiconductor laser," IEEE Journal of Quantum Electronics, vol. 17, no. 5, pp. 681-689, 1981.

[14] K. Otsuka and S. Tarucha, "Theoretical studies on injection locking and injection-induced modulation of laser diodes," IEEE Journal of Quantum Electronics, vol. 17, no. 8, pp. 1515-1521, 1981.

[15] G. H. M. van Tartwijk and D. Lenstra, "Semiconductor lasers with optical injection and feedback," Quantum and Semiclassical Optics, vol. 7, no. 2, pp. 87-143, 1995.

[16] R. Lang, "Injection locking properties of a semiconductor laser," IEEE Journal of Quantum Electronics, vol. 18, no. 6, pp. 976 -983, 1982. 
[17] C. H. Henry, N. A. Olsson, and N. K. Dutta, "Locking range and stability of injection locked $1.54 \mu \mathrm{m}$ InGaAsP," IEEE Journal of Quantum Electronics, vol. 21, no. 8, pp. 1152-1156, 1985.

[18] F. Mogensen, H. Olesen, and G. Jacobsen, "Locking conditions and stability properties for a semiconductor laser with external light injection," IEEE Journal of Quantum Electronics, vol. 21, no. 7, pp. 784-793, 1985.

[19] P. Gallion, H. Nakajima, G. Debarge, and C. Chabran, "Contribution of spontaneous emission to the linewidth of an injection-locked semiconductor laser," Electronics Letters, vol. 21, no. 14, pp. 626-628, 1985.

[20] N. Schunk and K. Petermann, "Noise analysis of injection-locked semiconductor injection lasers," IEEE Journal of Quantum Electronics, vol. 22, no. 5, pp. 642-650, 1986.

[21] P. Spano, S. Piazzolla, and M. Tamburrini, "Frequency and intensity noise in injection-locked semiconductor lasers: theory and experiments," IEEE Journal of Quantum Electronics, vol. 22, no. 3, pp. 427-435, 1986.

[22] I. Petitbon, P. Gallion, G. Debarge, and C. Chabran, "Locking bandwidth and relaxation oscillations of an injection-locked semiconductor laser," IEEE Journal of Quantum Electronics, vol. 24, no. 4, pp. 148 -154, 1988.

[23] O. Lidoyne, P. B. Gallion, and D. Erasme, "Modulation properties of an injection-locked semiconductor laser," IEEE Journal of Quantum Electronics, vol. 27, no. 3, pp. $344-351,1991$.

[24] J. Wang, M. K. Haldar, L. Li, and F. Mendis, "Enhancement of modulation bandwidth of laser diodes by injection locking," IEEE Photonics Technology Letters, vol. 8, no. 1, pp. 34-36, 1996.

[25] S. Wieczorek, W. W. Chow, L. Chrostowski, and C. J. Chang-Hasnain, "Improved semiconductor-laser dynamics from induced population pulsation," IEEE Journal of Quantum Electronics, vol. 42, no. 5-6, pp. 552-562, 2006

[26] L. Li, "Static and dynamic properties of injection-locked semiconductor lasers," IEEE Journal of Quantum Electronics, vol. 30, no. 8, pp. 1701 -1708, 1994

[27] O. Lidoyne, P. Gallion, C. Chabran, and G. Debarge, "Locking range, phase noise and power spectrum of an injection-locked semiconductor laser," IEE Proceedings, vol. 137, no. J3, pp. 147 -154, 1990

[28] M. B. Spencer and W. E. Lamb Jr., "Laser with a transmitting window," Physical Review A, vol. 5, no. 2, pp. 884-892, 1972.

[29] G. R. Hadley, "Injection locking of diode lasers," IEEE Journal of Quantum Electronics, vol. 22, no. 3, pp. 419-426, 1986.

[30] F. Favre, "Theoretical analysis of external optical feedback on DFB semiconductor lasers," IEEE Journal of Quantum Electronics, vol. 23, no. 1 , pp. $81-88,1987$.

[31] B. Tromborg, H. Olesen, X. Pan, and S. Saito, “Transmission line description of optical feedback and injection locking for Fabry-Perot and DFB lasers," IEEE Journal of Quantum Electronics, vol. 23, no. 11, pp. 1875 - 1889, 1987.

[32] R. Hui, A. D’Ottavi, A. Mecozzi, and P. Spano, “Injection locking in distributed feedback semiconductor lasers,” IEEE Journal of Quantum Electronics, vol. 27, no. 6, pp. 1688-1695, 1991

[33] J.-P. Bouyer and C. Bréant, "Stability of an injection-locked DFB $1.5 \mu \mathrm{m}$ semiconductor laser," Journal de physique III, vol. 9, pp. $1623-1644,1992$.

[34] J. Troger, P.-A. Nicati, L. Thévenaz, and P. A. Robert, "Novel measurement scheme for injection-locking experiments," IEEE Journal of Quantum Electronics, vol. 25, no. 6, pp. 1254-1260, 1989

[35] P. Vankwikelberge, G. Morthier, and R. Baets, "CLADISS - a longitudinal multimode model for the analysis of the static, dynamic, and stochastic behavior of diode lasers with distributed feedback," IEEE Journal of Quantum Electronics, vol. 26, no. 10, pp. 1728-1741, 1990.

[36] S. Hansmann, "Transfer matrix analysis of the spectral properties of complex distributed feedback laser structures," IEEE Journal of Quantum Electronics, vol. 28, no. 11, pp. 2589-2595, 1992.

[37] O. Hess and T. Kuhn, "Maxwell-Bloch equations for spatially inhomogeneous semiconductor lasers. i. theoretical formulation," Physical Review A, vol. 54, no. 4, pp. 3347-3359, 1996.

[38] — - "Maxwell-Bloch equations for spatially inhomogeneous semiconductor lasers. ii. spatiotemporal dynamics," Physical Review A, vol. 54, no. 4, pp. 3360-3368, 1996.

[39] M. Radzianus, H.-J. Wunsche, B. Sartorius, O. Brox, D. Hoffmann, K. Schneider, and D. Marcenac, "Modeling self-pulsating DFB lasers with an integrated phase tuning section,” IEEE Journal of Quantum Electronics, vol. 36, no. 9, pp. 1026-1034, 2000.

[40] U. Bandelow, M. Radziunas, J. Sieber, and M. Wolfrum, "Impact of gain dispersion on the spatio-temporal dynamics of multisection lasers," IEEE Journal of Quantum Electronics, vol. 37, no. 2, pp. 183-188, 2001 
[41] C. H. Henry, "Theory of spontaneous emission noise in open resonators and its application to lasers and optical amplifiers," Journal of Lightwave Technology, vol. 4, no. 3, pp. 288-297, 1986.

[42] T. Makino, "Transfer-matrix analysis of the intensity and phase noise of multisection DFB semiconductor lasers," IEEE Journal of Quantum Electronics, vol. 27, no. 11, pp. 2404-2414, 1991.

[43] R. Marani and M. Lax, "Spontaneous emission in non-hermitian optical-systems - distributed-feedback semiconductor-lasers," Physical Review A, vol. 52, no. 3, pp. 2376-2387, 1995.

[44] M. F. Peirera Jr and K. Henneberger, "Green's functions theory for semiconductor-quantum-well laser spectra," Physical Review B, vol. 53, no. 24, pp. 16485-16496, 1996.

[45] H. Wenzel, "Green's function based simulation of the optical spectrum of multisection lasers," IEEE Journal of Selected Topics in Quantum Electronics, vol. 9, no. 3, pp. 865-871, 2003.

[46] R. Loudon, D. Ramoo, and M. J. Adams, “Theory of spontaneous emission noise in multisection semiconductor lasers," Journal of Lightwave Technology, vol. 23, no. 8, pp. 2491-2504, 2006.

[47] B. Tromborg, H. Olesen, and X. Pan, “Theory of linewidth for multielectrode laser diodes with spatially distributed noise sources," IEEE Journal of Quantum Electronics, vol. 27, no. 2, pp. 178-192, 1991.

[48] X. Pan, H. Olesen, and B. Tromborg, "Modulation characteristics of tunable DFB/DBR lasers with one or two passive tuning sections," IEEE Journal of Quantum Electronics, vol. 25, no. 6, pp. 1254-1260, 1989.

[49] H. Olesen, B. Tromborg, X. Pan, and H. E. Lassen, "Stability and dynamic properties of multi-electrode laser diodes using Green's function approach," IEEE Journal of Quantum Electronics, vol. 29, no. 8, pp. 2282-2301, 1993.

[50] C. H. Chen and G. Griffel, "Static, dynamic, and noise analysis of multisection DFB lasers using frequency-domain transmission line model," IEEE Journal of Quantum Electronics, vol. 34, no. 9, pp. 1533-1544, 1998.

[51] B. Tromborg and J. Mørk, "Nonlinear injection locking dynamics and the onset of coherence collapse in external cavity lasers," IEEE Journal of Quantum Electronics, vol. 26, no. 4, pp. 642-654, 1990.

[52] G.-H. Duan, P. Gallion, and G. P. Agrawal, "Dynamic and noise properties of tunable multielectrode semiconductor lasers including spatial hole burning and nonlinear gain,” IEEE Journal of Quantum Electronics, vol. 29, no. 3, pp. 844-854, 1993.

[53] F. Grillot, B. Thedrez, and G.-H. Duan, "Feedback sensitivity and coherence collapse threshold of semiconductor dfb lasers with complex structures," IEEE Journal of Quantum Electronics, vol. 40, no. 3, pp. 231 - 240, 2004.

[54] E. Detoma, B. Tromborg, and I. Montrosset, "The complex way to laser diode spectra: example of an external cavity laser strong optical feedback," IEEE Journal of Quantum Electronics, vol. 41, no. 2, pp. 171-182, 2005.

[55] L. D. Landau and E. M. Lifshitz, Statistical physics. London:Addison Wesley Publishing Co., 1969.

[56] G. P. Agrawal and N. K. Dutta, Semiconductors lasers. Van Nostrand Reinhold, 1993.

[57] X. Pan, H. Olesen, and B. Tromborg, "Spectral linewidth of DFB lasers including the effects of spatial holeburning and nonuniform current injection,” IEEE Photonics Technology Letters, vol. 2, no. 5, pp. 312-315, 1990.

[58] G. P. Agrawal, "Gain nonlinearities in semiconductor lasers: theory and application to distributed feedback lasers," IEEE Journal of Quantum Electronics, vol. 23, no. 6, pp. 860-868, 1987.

[59] K. Petermann, "Calculated spontaneous emission factor for double-heterostructure injection lasers with gain-induced waveguiding," IEEE Journal of Quantum Electronics, vol. 15, no. 7, pp. 566-570, 1979.

[60] C. Henry and R. F. Kazarinov, "Quantum noise in photonics," Review of Modern Physics, vol. 68, no. 3, pp. 802 - $851,1996$.

[61] M. Lax, "Classical noise IV:Langevin methods," Review of Modern Physics, vol. 38, pp. 541-566, 1966.

[62] J. Genest, M. Chamberland, P. Tremblay, and M. Têtu, "Microwave signals generated by optical heterodyne between injection-locked semiconductor lasers," IEEE Journal of Quantum Electronics, vol. 33, no. 6, pp. 989-998, 1997.

[63] P. Spano, S. Piazzolla, and M. Tamburrini, "Phase noise in semiconductor lasers: a theoretical approach," IEEE Journal of Quantum Electronics, vol. 19, no. 7, pp. $1195-1199,1994$.

[64] B. J. Thedrez and C. H. Lee, "Reassessment of standard rate equations for low facet reflectivity semiconductor lasers using traveling wave rate equations," IEEE Journal of Quantum Electronics, vol. 28, no. 12, pp. 2706-2713, 1992.

[65] X. Wang and N. J. Gomes, "Locking bandwidth equations for electrically and optically injection-locked oscillators," IEE ProceedingsOptoelectronics, vol. 151, no. 6, pp. 476-481, 2004. 
[66] K. Kurokawa, "Noise in synchronized oscillators," IEEE Transactions on microwave theory and techniques, vol. 16, no. 4, pp. 234-240, 1968.

[67] R. Adler, "A study of locking phenomena in oscillators," in Proceedings IRE, vol. 34, 1946, pp. 351-356.

[68] J. Mink and B. H. Verbeek, "Asymetric noise and output power in semiconductor lasers with optical feedback near threshold," Applied Physics Letters, vol. 48, no. 12, pp. 745-747, 1986. 